\title{
The Decay Lifetime of Polarized Fermions in Flight
}

\author{
Zhi-qiang Shi * \\ Faculty of Science, Xi'an Jiaotong University, Xi'an 710049, China \\ Guang-jiong $\mathrm{Ni}^{\dagger}$ \\ Department of Physics, Fudan University, Shanghai, 200433, P.R.China \\ Department of Physics, Portland State University, Portland, OR 97207 USA.
}

\begin{abstract}
Based on the parity violation in Standard model, we study the dependence of lifetime on the helicity of an initial-state fermion in weak interactions. It is pointed out that if the initial fermions in the decays are longitudinally polarized, then the decay lifetime of left-handed polarized fermions is different from that of right-handed polarized fermions in flight with a same velocity in a same inertial system.

PACS numbers: 11.30.Er; 11.30.Rd;12.15.-y; 13.30.-a; 13.35.Bv
\end{abstract}

The phenomena of parity violation are experimentally exhibited in two aspects. (1) The angular distribution of the particles emitted from decay processes is asymmetric. The experiment has established that the emission of beta particles is more favored in the direction opposite to that of the ${ }^{60} \mathrm{Co}$ nuclei spin.[1] In muon decays, this asymmetry has also been observed.[2] The parity violation in the $\Lambda$-decay is manifested as an up-down asymmetry of the decay pion (or proton) relative to the production plane. It is worthy of special mention that the asymmetry of the angular distribution is dependent on the spin orientation of the initial decay particle. If the average spin component of the initial particle is equal to zero, the angular distribution is symmetric. (2) All of fermions emitted from decay processes are longitudinally polarized. It is well known that the neutrinos are left-handed (LH) polarized while the antineutrinos are right-handed $(\mathrm{RH})$ polarized. In beta or muon decays it is found that the electrons are always LH polarized while the positrons are RH polarized.[3] Based on the two experimental facts above, naturally, it is thought that not only the final-state fermions in the decays, but also the initial-state fermion should be relevant to its longitudinal polarization. So we further could consider that the lifetime of fermions in the LH helicity state and the that of fermions in the RH helicity state should be different as well. However, The experimental and theoretical study on the polarization or helicity of the initial fermions in decays has not yet been discussed fully in the literature. In this paper we aim at exploring this point in some detail.

${ }^{*}$ Present address: Residence 10-2-7, Shaanxi Normal University, Xi'an 710062, China. E-mail address: zqshi@snnu.edu.cn

${ }^{\dagger}$ E-mail address: gj_ni@Yahoo.com 


\section{The charged weak currents in the SM}

In order to describe the parity violation in weak interactions, all of fundamental fermions are divided into two classes, LH chirality state and $\mathrm{RH}$ chirality state, in the standard model (SM). They are defined as

$$
\psi_{L}=\frac{1}{2}\left(1+\gamma_{5}\right) \psi, \quad \psi_{R}=\frac{1}{2}\left(1-\gamma_{5}\right) \psi
$$

respectively. And we have

$$
\psi=\psi_{L}+\psi_{R} .
$$

The LH chirality state is different from the RH chirality state. The former is the SU(2)-doublet state whereas the latter the SU(2)-singlet state, hence they have different gauge transformations. Especially, RH chirality state has zero weak isospin and is only present in neutral weak currents. Therefore, there exist the only LH chirality states in charged weak currents. The interaction Lagrangians for charged weak lepton current and charged weak quark current read, respectively

$$
\begin{aligned}
& \mathcal{L}_{\ell W}=\frac{1}{\sqrt{2}} g_{2} \bar{e}_{L} \gamma_{\mu} W_{\mu}^{+} \nu_{L}+\frac{1}{\sqrt{2}} g_{2} \bar{\nu}_{L} \gamma_{\mu} W_{\mu}^{-} e_{L} \\
& \mathcal{L}_{q W}=\frac{1}{\sqrt{2}} g_{2} \bar{d}_{L} \gamma_{\mu} W_{\mu}^{+} u_{L}+\frac{1}{\sqrt{2}} g_{2} \bar{u}_{L} \gamma_{\mu} W_{\mu}^{-} d_{L},
\end{aligned}
$$

where $g_{2}$ is the coupling constant corresponding to $\mathrm{SU}(2)$. Obviously, all of fermions are in the LH chirality states and all of antifermions while in the RH chirality states in decay processes.

For example, the weak interaction in muon decay is successfully described by four-fermion interaction Hamiltonian. We denote the matrix element by

$$
M \sim \sum g_{\varepsilon \mu}^{\gamma}\left\langle\bar{e}_{\varepsilon}\left|\Gamma_{\gamma}\right|\left(\nu_{e}\right)_{n}\right\rangle\left\langle\left(\bar{\nu}_{\mu}\right)_{m}\left|\Gamma_{\gamma}\right| \mu_{\mu}\right\rangle,
$$

where $\gamma=S, V, T$ indicates a scalar, vector or tensor interaction; and $\varepsilon, \mu=R, L$ indicate a right- or left-handed chirality of the electron or muon. The chiralities $n$ and $m$ of the $\nu_{e}$ and $\bar{\nu}_{\mu}$ are then determined by the values of $\gamma, \varepsilon$ and $\mu$. All the coupling constants have been obtained entirely from experiments without any model assumption. [4] The experiments on muon decay show $g_{R L}, g_{R R}, g_{L R}$ to be zero, and at least one of the two coupling, $g_{L L}^{V}$ or $g_{L L}^{S}$, to be nonzero. The experiments on inverse muon decay provides a lower limit for pure $V-A$ interaction with $\left|g_{L L}^{V}\right|>0.960$. Thus the measurements give a strong support to the standard model which

sets $g_{L L}^{V}=1$ and all the others being zero, and then indicate that the charged weak current is dominated by a coupling to left-handed chirality fermions. Therefore, the negative muon decay can be written as

$$
\mu_{L}^{-} \longrightarrow e_{L}^{-}+\bar{\nu}_{e_{R}}+\nu_{\mu_{L}} .
$$

And the matrix element (5) has the form

$$
M=\frac{g_{2}}{4 m_{W}^{2}}\left(\bar{\nu}_{\mu_{L}} \gamma_{\mu} \mu_{L}\right)\left(\bar{e}_{L} \gamma_{\mu} \nu_{e_{L}}\right)
$$

Similarly, the neutron decay can be written as

$$
n_{L} \longrightarrow p_{L}+e_{L}+\bar{\nu}_{R}
$$


and the corresponding matrix element is

$$
M=\frac{g_{2}}{4 m_{W}^{2}}\left(\bar{p}_{L} \gamma_{\mu} n_{L}\right)\left(\bar{e}_{L} \gamma_{\mu} \nu_{e_{L}}\right)
$$

\section{Helicity and chirality}

The Dirac equation has the form in Pauli metric

$$
\left(\gamma_{\mu} \partial_{\mu}+m c^{2}\right) \psi=0
$$

where $m$ is the rest mass and $\psi$ is four-component spinor. The projection of spin vector $\vec{\sigma}$ along the direction of fermion momentum is known as the helicity or polarization:

$$
h=\frac{\vec{\sigma} \cdot \vec{p}}{|\vec{p}|}
$$

where $h$ is a constant of the motion with eigenvalues \pm 1 . Taking the simplest case of $\vec{p}: p_{z}=p$, when $h=+1$ and $h=-1$, we have the RH helicity state $\psi_{R h}$ and the LH helicity state $\psi_{L h}$, respectively

$$
\psi_{R h}=\left(\begin{array}{c}
1 \\
0 \\
\frac{p c}{E+m c^{2}} \\
0
\end{array}\right) e^{\frac{i}{\hbar}(p z-E t)}, \quad \psi_{L h}=\left(\begin{array}{c}
0 \\
1 \\
0 \\
\frac{-p c}{E+m c^{2}}
\end{array}\right) e^{\frac{i}{\hbar}(p z-E t)}
$$

Setting

$$
\psi=\left(\begin{array}{c}
\varphi \\
\chi
\end{array}\right)
$$

where $\varphi$ and $\chi$ are two-component spinors, we may rewrite (10) as a set of two coupled equations for $\varphi$ and $\chi$ :

$$
\left\{\begin{array}{l}
\vec{\sigma} \cdot \vec{p} c \chi=\left(E-m c^{2}\right) \varphi \\
\vec{\sigma} \cdot \vec{p} c \varphi=\left(E+m c^{2}\right) \chi
\end{array}\right.
$$

We have then

$$
\varphi=\frac{\vec{\sigma} \cdot \vec{p} c}{E-m c^{2}} \chi, \quad \chi=\frac{\vec{\sigma} \cdot \vec{p} c}{E+m c^{2}} \varphi
$$

On the other hand, $\psi_{L}$ and $\psi_{R}$ are the eigenfunctions of the chirality operator $\gamma_{5}$, satisfying respectively

$$
\gamma_{5} \psi_{L}=\psi_{L}, \quad \gamma_{5} \psi_{R}=-\psi_{R} .
$$

For describing the relation between the chirality state and the helicity state, we introduce two linear combination function of $\varphi$ and $\chi$

$$
\phi_{R}=\frac{1}{\sqrt{2}}(\varphi+\chi), \quad \phi_{L}=\frac{1}{\sqrt{2}}(\varphi-\chi) .
$$

Then

$$
\varphi=\frac{1}{\sqrt{2}}\left(\phi_{R}+\phi_{L}\right), \quad \chi=\frac{1}{\sqrt{2}}\left(\phi_{R}-\phi_{L}\right) .
$$


Substituting into formula (13), we get

$$
\psi=\left(\begin{array}{c}
\varphi \\
\chi
\end{array}\right)=\frac{1}{\sqrt{2}}\left(\begin{array}{c}
\phi_{L} \\
-\phi_{L}
\end{array}\right)+\frac{1}{\sqrt{2}}\left(\begin{array}{c}
\phi_{R} \\
\phi_{R}
\end{array}\right) .
$$

Comparing with Eq.(2) this gives

$$
\psi_{L}=\frac{1}{\sqrt{2}}\left(\begin{array}{c}
\phi_{L} \\
-\phi_{L}
\end{array}\right), \quad \psi_{R}=\frac{1}{\sqrt{2}}\left(\begin{array}{c}
\phi_{R} \\
\phi_{R}
\end{array}\right) .
$$

Hence, instead of Eq.(14), we find the Dirac equation in Weyl (chiral) representation:

$$
\left\{\begin{array}{l}
(E-\vec{\sigma} \cdot \vec{p} c) \phi_{R}=m c^{2} \phi_{L} \\
(E+\vec{\sigma} \cdot \vec{p} c) \phi_{L}=m c^{2} \phi_{R}
\end{array}\right.
$$

Obviously, both $\phi_{L}$ and $\phi_{R}$ are not the eigenfunction of helicity operator $h$. When $m=0$ or $E \gg m$, however, they are the eigenfunction of helicity operator $h$. For example, because neutrino has zero mass, the helicity state of neutrino is identical with its chirality state.

In general, for $h=-1$, we obtain from Eq.(21)

$$
\left|\frac{\phi_{R}}{\phi_{L}}\right|_{L h}=\frac{m c^{2}}{E+p c}=\sqrt{\frac{c-v}{c+v}}=W,
$$

where $v$ is the fermion velocity and $W$ is the Weyl parameter introduced in [5]. Once $v>0$, a LH polarized fermion has $h=-1$ explicitly and $\phi_{L}>\phi_{R}$ implicitly. One can see that the hidden amplitude of RH chirality state decreases with the increases of $v$ until $\phi_{R} \rightarrow 0$ when $v \rightarrow c$, showing that a high-energy fermion can be LH polarized without hidden RH spinning instability. With $v=0$, we have $\phi_{R}=\phi_{L}$, i.e. the hidden amplitude of LH chirality state is equal to that of RH chirality state and one even can not discriminate its polarization being $\mathrm{LH}$ or $\mathrm{RH}[6]$.

Similarly, for $h=+1$, we obtain

$$
\left|\frac{\phi_{R}}{\phi_{L}}\right|_{R h}=\frac{m c^{2}}{E-p c}=\sqrt{\frac{c+v}{c-v}}=\frac{1}{W} .
$$

Once $v>0$, a RH polarized fermion has $h=1$ explicitly and $\phi_{R}>\phi_{L}$ implicitly. The latter decreases with the increase of $v$ until $\phi_{L} \rightarrow 0$ when $v \rightarrow c$, showing that a high-energy fermion can be RH polarized without hidden LH spinning instability.

\section{The lifetime of polarized fermions}

Usually, in calculating the decay probability $\Gamma$ of a fermion (muon or neutron) in weak interactions, one defined the lifetime $\tau=1 / \Gamma$ with $\Gamma$ being that of fermion at rest and its spin direction being averaged. So no discrimination between helicities was involved. Now we will take the velocity dependence of $\Gamma$ for polarized fermion into account. To this purpose, the absolute square of spinor $\psi$ reads

$$
|\psi|^{2}=\left|\phi_{L}\right|^{2}+\left|\phi_{R}\right|^{2}=\left|\phi_{L}\right|^{2}\left(1+\frac{\left|\phi_{R}\right|^{2}}{\left|\phi_{L}\right|^{2}}\right)
$$


Which leads to

$$
\left|\phi_{L}\right|^{2}=\frac{|\psi|^{2}}{1+\left|\frac{\phi_{R}}{\phi_{L}}\right|^{2}}
$$

Since the charged weak current originates from the left-handed chirality state only, from Eqs. (3) and (4) or the matrix element (7) and (9), we get the ratio of $\left|\phi_{L}\right|^{2}$ in a RH helicity state to that in a LH helicity state, yielding the relevant ratio of decay probability for moving fermion in weak interactions,

$$
\frac{\Gamma_{R}}{\Gamma_{L}}=\frac{\left|\phi_{L}\right|_{R h}^{2}}{\left|\phi_{L}\right|_{L h}^{2}}=\frac{1+W^{2}}{1+\frac{1}{W^{2}}}=W^{2}=\frac{c-v}{c+v} .
$$

From

$$
\Gamma=\frac{1}{2}\left(\Gamma_{R}+\Gamma_{L}\right) \text { and } \quad \Gamma=\frac{\hbar}{\tau},
$$

we find the RH polarized fermion lifetime $\tau_{R}$ and LH polarized fermion lifetime $\tau_{L}$, respectively

$$
\tau_{R}=\tau \frac{c}{c-v} \quad \text { and } \quad \tau_{L}=\tau \frac{c}{c+v}
$$

where $\tau$ is the average lifetime. According to the theory of special relativity, we may write $\tau$ as

$$
\tau=\frac{\tau_{0}}{\sqrt{1-(v / c)^{2}}}
$$

where $\tau_{0}$ is the lifetime of fermion at rest. Therefore

$$
\tau_{R}=\frac{\tau_{0}}{\sqrt{1-(v / c)^{2}}} \frac{c}{c-v} \quad \text { and } \quad \tau_{L}=\frac{\tau_{0}}{\sqrt{1-(v / c)^{2}}} \frac{c}{c+v} .
$$

It is easy to see that the $\tau_{R}$ is greater than $\tau_{L}$, which shows the lifetime asymmetry of leftright handed polarized fermion. The lifetime asymmetry is simply expressed by the familiar parameter $\beta$ in special relativity

$$
\text { lifetime asymmetry } \equiv \frac{\tau_{R}-\tau_{L}}{\tau_{R}+\tau_{L}}=\beta=\frac{v}{c} \text {. }
$$

When $v=0$, we find

$$
\tau_{R}=\tau_{L}=\tau_{0}, \quad(\beta=0)
$$

When $v \rightarrow c$, we find

$$
\tau_{R} \rightarrow \infty, \quad \tau_{R} \rightarrow \infty, \quad(\beta \rightarrow 1) .
$$

In particular, when $v=\frac{1}{2} c, \beta=\frac{1}{2}$, the lifetime of the LH polarized fermions has a minimum value $\tau_{L}=\tau_{\min }=0.77 \tau_{0}$.

It is not difficult to prove that the lifetimes of antifermions in motion are given by

$$
\bar{\tau}_{R}=\frac{\tau_{0}}{\sqrt{1-(v / c)^{2}}} \frac{c}{c+v} \text { and } \quad \bar{\tau}_{L}=\frac{\tau_{0}}{\sqrt{1-(v / c)^{2}}} \frac{c}{c-v} .
$$




\section{Summary and discussion}

The Dirac equation is invariant under a space reflection $\vec{x} \rightarrow-\vec{x}$, provided the spinor field $\psi(\vec{x}, t)$ undergoes the corresponding transformation

$$
\psi(\vec{x}, t) \rightarrow \psi(-\vec{x}, t)=\gamma_{4} \psi(\vec{x}, t)
$$

Applying (35) to (1) we get

$$
\left\{\begin{array}{l}
\psi_{L}(\vec{x}, t)=\frac{1}{2}\left(1+\gamma_{5}\right) \psi(\vec{x}, t) \rightarrow \frac{1}{2}\left(1+\gamma_{5}\right) \psi(-\vec{x}, t)=\frac{1}{2}\left(1+\gamma_{5}\right) \gamma_{4} \psi(\vec{x}, t)=\gamma_{4} \psi_{R}(\vec{x}, t), \\
\psi_{R}(\vec{x}, t)=\frac{1}{2}\left(1-\gamma_{5}\right) \psi(\vec{x}, t) \rightarrow \frac{1}{2}\left(1-\gamma_{5}\right) \psi(-\vec{x}, t)=\frac{1}{2}\left(1+\gamma_{5}\right) \gamma_{4} \psi(\vec{x}, t)=\gamma_{4} \psi_{L}(\vec{x}, t) .
\end{array}\right.
$$

It means that despite the invariance of Dirac equation under transformation (35), the LH and $\mathrm{RH}$ chirality components of $\psi$ transform each other. Alternatively, it would be better to consider the space inversion transform here in terms of two-component spinors $\phi_{L}$ and $\phi_{R}$ regardless of the theory being invariant or not after the following transformation:

$$
\left\{\begin{array}{l}
\phi_{L}(\vec{x}, t) \rightarrow \phi_{L}(-\vec{x}, t) \rightarrow \phi_{R}(\vec{x}, t) \\
\phi_{R}(\vec{x}, t) \rightarrow \phi_{R}(-\vec{x}, t) \rightarrow \phi_{L}(\vec{x}, t)
\end{array}\right.
$$

It is easy to see that under the space inversion the Dirac equations (21) is invariant, showing that the law of motion of a Dirac particle preserves the parity symmetry. On the other hand, the equations (28) and (34) are also invariant, respectively, under a space reflection and the transformation (37). It means that the $\tau_{L}$ is always smaller than $\tau_{R}$ for fermions in flight and the $\tau_{R}$ is always smaller than $\tau_{L}$ for antifermions in any one of inertial systems. It is just because only the LH chirality components $\left(\phi_{L}\right)$ of fermions participates in the decay process whereas their RH chirality components $\left(\phi_{R}\right)$ do not. For example, under the space inversion (37), a moving fermion with $h=-1$ having $\phi_{L}>\phi_{R}$ and $\tau_{L}=\tau /(1+\beta)$ will transform into a fermion moving in opposite direction with $h=+1$ having $\phi_{R}>\phi_{L}$ and $\tau_{R}=\tau /(1-\beta)$. Hence the property that the lifetime $\tau_{L}$ of the LH polarized fermion is shorter than the lifetime $\tau_{R}$ of the $\mathrm{RH}$ polarized fermion in flight ( with a same speed in the same inertial system ) shows a serious violation of parity symmetry. More intuitively, since a neutron having a spin $\vec{S}_{n}$ will emit an electron $e^{-}$and an antineutrino $\bar{\nu}_{e}$ basically in the backward direction to $\vec{S}_{n}$, we may imagine a decaying neutron being like a "comet" with its head oriented to $\vec{S}_{n}$ and its tail composed of $e^{-}$ and an $\bar{\nu}_{e}$. Obviously, this neutron is by no means an isotropic object in $\beta$ decay. Rather, it is just like a highly anisotropic "comet". A space inversion would transform it into an unexisting

(ridiculous) "comet" with its tail parallel to $\vec{S}_{n}$, showing the parity violation. Moreover, if we push the rest "comet" into motion either along with or opposite to the direction of $\vec{S}_{n}$, we will find left-right asymmetry of the lifetime in its decay, showing $\tau_{L}<\tau_{R}$ with the same speed. To our understanding, it is just this strange phenomenon relevant to the parity violation that was overlooked in the past.

As seen from formula (31), the lifetime asymmetry $\beta$ increases with $v$ increases, and is negligible at low energy. In neutron decay, for example, $\beta=0.046$ when neutron kinetic energy $T=1 \mathrm{MeV}$, and $\beta=0.145$ when $T=10 \mathrm{MeV}$. Published neutron lifetime were measured using thermal or cold neutron beams.[7] The energy of these beams is below $1 \mathrm{eV}$, and too 
small for the lifetime asymmetry effect to be observe. One actually lacks experimental evidence in support of this rather natural consequence of the SM or pure V-A theory. In view of the important implications of these observations, we report them here now in the hope that they may stimulate and encourage further experimental investigations on the question of polarized fermion lifetime in either beta or muon decays.

Since $\tau_{R}>\tau_{L}$, an unpolarized neutron beams would gradually turn into RH polarized in propagation process. Due to neutron decay, according to formula (8), the amount of LH protons and LH electrons would be predominant in the final state. In fact, as early as 1956 T.D.Lee and C.N.Yang pointed out: "There must exist two kinds of protons $p_{R}$ and $p_{L}$, the right-handed one and the left-handed one. Furthermore, at the present time the protons in laboratory must be predominantly of one kind in order to produce the supposedly observed asymmetry". [8] If the lifetime asymmetry of polarized fermion in flight described above is correct, one kind of helicity fermions would be more abundant than another kind in some region of the universe. The measured polarized fermion lifetime may be crucial for further understanding of the weak interactions and there might be important consequences for cosmology and astrophysics. Especially, it would be closely related to various neutrino anomalies.

\section{References}

[1] C.S.Wu, E.Amblere, R.W.Hayward, D.D.Hoppes and R.P.Hudson, Phys. Rev. 105 $1413(1957)$.

[2] R.L.Garwin, L.M.Lederman and M.Weinrich, Phys. Rev. 105, 1415(1957); J.I.Friedman and V.L.Telegdi, Phys. Rev. 105, 1681(1957).

[3] H.Frauenfelder, R.Bobone, E.V.Goeler, et al., Phys. Rev. 106, 386(1957); Particle Data Group, The Europ. Phys. J. C15, 318(2000); D.H.Perkins, Introduction to High Energy Physics, Addison-Wesley Publishing Company, Inc., 1972, pp.135-150.

[4] Y. Kuno and Y. Okada, Rev. Mod. Phys. 73, 151(2001); Particle Data Group, The Europ. Phys. J. C, 317(2000); W. Fetscher, H.-J. Gerber, and K. F. Johnson, Phys. Lett. B 173, 102(1989); CHARM II Collaboration, P. Vilain et al., Phys. lett. B 364, 121(1995).

[5] G.-J.Ni and T.Chang, Preprint, hep-ph/0103051.

[6] L.H.Ryder, Quantum Field Theory, Cambrige, Cambrige University Press, 1996.

[7] J.Last, M.Arnold, J.Döhner, et al., Phys.Rev.Lett 60,995(1988).

[8] T.D.Lee and C.N.Yang, Phys. Rev. 104(1), 254(1956). 